\title{
Promoting functions of microRNA-29a/199B in neurological recovery in rats with spinal cord injury through inhibition of the RGMA/STAT3 axis
}

Weijie Yang and Ping Sun ${ }^{*}$ (1)

\begin{abstract}
Background: The prognostic and therapeutic potential of microRNAs (miRNAs) in spinal cord injury (SCl) has aroused increasing concerns. This study aims to research the functions of miR-29a/199B in the neurological function recovery after $\mathrm{SCl}$ and the mechanical mechanism.

Methods: A rat model with $\mathrm{SCl}$ was induced with sham-operated ones as control. The locomotor function and coordination of rat hindlimbs were determined by a Basso, Beattie, and Bresnahan (BBB) locomotor rating scale and a ladder-climbing test, respectively. Expression of a neurofilament protein NF-200 and synaptophysin in gray matter of rats was determined to evaluate neuronal recovery in a cellular perspective. Binding relationships between miR29a/199B with RGMA were predicted and validated using luciferase assays. Altered expression of miR-29a/199B and RGMA was introduced to explore their functions in rat neurological functions. The protein level and phosphorylation of STAT3 in gray matter were measured by western blot analysis.

Results: miR-29a and miR-199B were poorly expressed, while RGMA was abundantly expressed in gray matter at injury sites. Either miR-29a or miR-199B could bind to RGMA. Overexpression of miR-29a/199B or silencing of RGMA led to an increase in BBB locomotor scores, hindlimb coordination ability, and the expression of NF-200 and synaptophysin in gray matter. Further inhibition in miR-29a/199B blocked the promoting roles of RGMA silencing in neurological recovery. Upregulation of miR-29a/199B or downregulation of RGMA suppressed the phosphorylation of STAT3.
\end{abstract}

Conclusion: This study evidenced that miR-29a and miR-199B negatively regulated RGMA to suppress STAT3 phosphorylation, therefore promoting the neurological function recovery in rats following SCl.

Keywords: MicroRNA-29a; MicroRNA-199B, RGMA, Spinal cord injury, STAT3 signaling pathway; Neurological recovery

\footnotetext{
*Correspondence: Sunping4231@163.com

Department of Orthopedics, Shanghai Eighth People's Hospital, No.8, Caobao

Road, Shanghai 200235, People's Republic of China
}

(c) The Author(s). 2020 Open Access This article is licensed under a Creative Commons Attribution 4.0 International License, which permits use, sharing, adaptation, distribution and reproduction in any medium or format, as long as you give appropriate credit to the original author(s) and the source, provide a link to the Creative Commons licence, and indicate if changes were made. The images or other third party material in this article are included in the article's Creative Commons licence, unless indicated otherwise in a credit line to the material. If material is not included in the article's Creative Commons licence and your intended use is not permitted by statutory regulation or exceeds the permitted use, you will need to obtain permission directly from the copyright holder. To view a copy of this licence, visit http://creativecommons.org/licenses/by/4.0/ The Creative Commons Public Domain Dedication waiver (http://creativecommons.org/publicdomain/zero/1.0/) applies to the data made available in this article, unless otherwise stated in a credit line to the data. 


\section{Background}

Traumatic spinal cord injury (SCI) is usually caused by an external physical impact damage to the spinal cord and contributes to everlasting neurological impairment and dysfunction and burdens individuals, families, and the society with a high economic cost [1]. Even though survive the initial injury, the SCI patients face great risks of medical complications throughout their lives, which is often related to infectious morbidity, leading to significant reductions in life quality and expectancy [2]. Advanced therapies such as decompression, functional stimulation, neurorehabilitation, nerve and tendon transfers, and neural bypass, as well as drug treatments, surgery, and cell transplantation, are currently promising strategies for SCI, but there are no available regenerative treatments to date to cure the disease [3, 4]. Understanding of molecular biomarkers may help program candidacy and tailor treatment for patients [4], and therefore, identifying novel molecular biomarkers and mechanisms holds great potential to improve the outcome of SCI patients.

MicroRNAs (miRNAs) are reported as one of the important biomarkers associated with the injury severity and prognosis of $\mathrm{SCI}$, which may predict spontaneous neurological recovery over time with high precision, sensitivity, and reproducibility [5]. Thanks to their unique potent function in regulating target gene expression post-transcriptionally, miRNAs are emerging potential tools or targets for SCI treatment with great therapeutic significances [6, 7]. miR-29a and miR-199B have been noted to promote axonal regeneration and neuronal functional recovery or reduce the inflammatory response in SCI $[8,9]$, but their functions and implicated mechanisms in SCI progression are not fully studied yet. Interestingly, the online prediction on a bioinformatics system StarBase (http://starbase.sysu.edu.cn/) in our study predicted that these two miRNAs shared a binding relationship with the same target gene, repulsive guidance molecule A (RGMA). RGMA is a GPI-linked glycoprotein that exists in soluble and membrane-bound forms and suppresses neurite growth by binding to neogenin, its neuronal receptor [10]. Correspondingly, inhibition of either RGMA or neogenin was found to promote neuronal survival and functional recovery after SCI $[11,12]$. The signal transducer and activator of transcription 3 (STAT3) signaling is implicated in multiple inflammatory responses by regulating some proinflammatory cytokines such as interleukin-6 (IL-6) and IL-17, and its downregulation was found to reduce spinal cord neuroinflammation and promote neuronal functional recovery after SCI [13, 14]. In addition, STAT3 is expressed by neurons, astrocytes, and other cell types in the central nervous system (CNS), and it is significantly activated following CNS insults [15]. Taken the above discussion together, we speculated that miR29a/miR-199B possibly targets RGMA to relieve SCI. Therefore, a rat model with SCI was established $[16,17]$ to validate this hypothesis and to explore whether STAT3 is involved in the events.

\section{Materials and methods}

\section{Ethics statement}

The study was performed with the approval of the Ethical Committee of Shanghai Eighth People's Hospital (Approval No. 2019011). All experimental procedures were conducted in line with the ethical guidelines for the study of experimental pain in conscious animals. Great efforts were made to minimize the usage and suffering of animals.

\section{Establishment of a rat model with $\mathrm{SCI}$}

A total of 80 male Sprague-Dawley rats (8 weeks old, 200-220 g) purchased from the Experimental Animal Center of Sun Yat-sen University were housed in specific-pathogen-free (SPF) grade animal rooms with controlled humidity (60-65\%) and temperature (22$25^{\circ} \mathrm{C}$ ). The mice were allowed ad libitum feed and water and acclimated in a 12-h-dark/light cycle for 1 week. Thereafter, 72 rats were used for SCI model establishment. In brief, the rats were inhaled with $2 \%$ isoflurane and the mixture of Nitrous oxide and oxygen $(1: 2, \mathrm{v} / \mathrm{v})$ for anesthesia. Under a sterile condition, the T9-T11 laminae were removed by laminectomy, and then the T10 segment was compressed using a modified aneurysm clip at $20 \mathrm{~g}$ for $60 \mathrm{~s}$. Pain was alleviated by analgesics, while the infection was prevented with antibiotics. Successful model establishment in rats was confirmed by a $<1$ score $1 \mathrm{~h}$ after surgery according to a Basso, Beattie, and Bresnahan (BBB) locomotor rating scale $[18,19]$. The remaining 8 rats were subjected to sham operation with only the skin and muscle cut open and sutured.

After model establishment, each model rat was injected with $2.5 \mu \mathrm{L}$ lentiviral vector ( $\mathrm{LV})$ at $3 \mathrm{~mm}$ next to the lesion site. The needle was maintained for $5 \mathrm{~min}$ and quickly pulled out to avoid backflow. The injection was performed twice a week for a total of 6 weeks. Thereafter, the animals were euthanized through intraperitoneal injection of $150 \mathrm{mg} / \mathrm{kg}$ pentobarbital sodium and then the tissues from the gray matter in the lesion sites. The animals were allocated into the following groups after the corresponding injection: SCI group (model group without transfection), negative control (NC) mimic group $\left(7.5 \times 10^{5} \mathrm{TU}\right.$ LV-NC mimic), miR29a mimic (7.5 $\times 10^{5}$ TU LV-miR-29a mimic), miR199B mimic $\left(7.5 \times 10^{5}\right.$ TU LV-miR-199B mimic), short hairpin (sh)-NC group (7.5 $\times 10^{5} \mathrm{TU}$ LV-sh-NC), shRGMA (7.5 $\times 10^{5}$ TU LV-sh-RGMA), sh-RGMA + NC 
inhibitor group $\left(7.5 \times 10^{5}\right.$ TU LV-sh-RGMA + NC inhibitor), sh-RGMA + miR-29a inhibitor $\left(7.5 \times 10^{5} \mathrm{TU}\right.$ LV-sh-RGMA + miR-29a inhibitor), and sh-RGMA + miR-199B inhibitor $\left(7.5 \times 10^{5}\right.$ TU LV-sh-RGMA + miR199B inhibitor) ( $n=8$ in each group).

\section{Dual-luciferase reporter gene assay}

The binding relationships between miR-29a/199B and RGMA were predicted on StarBase. Then, the putative wild-type (WT) RGMA 3'UTR fragment was designed and inserted into pMIR-reporter plasmids (Promega, Madison, WI, USA) to construct pMIR-RGMA-WT vectors, and the corresponding mutant-type (MUT) binding sites between RGMA 3'UTR and miR-29a/199B were used to construct pMIR-RGMA-MUT vectors. Wellconstructed WT and MUT vectors were co-transfected with miR-29a/199B mimic or NC mimic into HEK293T cells (Beinuo Biotechnology Co., Ltd., Shanghai, China). Forty-eight hours later, the relative luciferase activity was measured on a Dual-Luciferase Reporter Assay System (Promega).

\section{Immunofluorescence staining}

The longitudinal sections of the spinal tissues were collected from rats on the 6th week. The sections were fixed in $40 \mathrm{~g} / \mathrm{L}$ paraformaldehyde for $15 \mathrm{~min}$, washed in phosphate buffer saline (PBS), and blocked in PBS containing $0.1 \%$ Triton $\mathrm{X}-100$ and $5 \%$ bovine serum albumin (BSA) at $37^{\circ} \mathrm{C}$ for $50 \mathrm{~min}$. Next, the slides were incubated with neurofilament 200 antibody (NF-200, 1: 200, \#55453, Cell Signaling Technology (CST), Beverly, MA, USA) at $4{ }^{\circ} \mathrm{C}$ overnight and then with cy3-labeled secondary antibody goat anti-rabbit immunoglobulin $\mathrm{G}$ (IgG) (ab6939, 1:500, Abcam Inc., Cambridge, MA, USA) at room temperature for $60 \mathrm{~min}$. Then, the slides were sealed and observed under a LSM510 meta confocal microscope (Carl Zeiss MicroImaging, Inc., Thornwood, NY, USA). The gray matter at the region $2-3 \mathrm{~mm}$ posterior to the spinal injury site was used for measurement. Relative NF-200-positive area was calculated using an Image J 2.0 software.

\section{BBB locomotor rating scores}

The hindlimb motor function of rats was evaluated using the BBB locomotor rating scale on $1,2,3,4,5$, and 6 weeks after model establishment, respectively. Briefly, the rats were placed in an open box and stimulated to move forward. The hip, knee, ankle flexibility, and the movement and coordination were evaluated. If the rats had isolated joint movements with no or little hindlimb movement, the rat would be allocated into an early stage (score $0-7$ ). Rats had occasional uncoordinated stepping would be classified into an intermediate stage (score 813). Rats that were capable of hindlimb coordination, equilibrium, and stepping were categorized into a late stage (score 14-21) [20].

\section{Ladder-climbing test}

Locomotion of animals was further determined using a ladder-climbing test. On 1, 2, 3, 4, 5, and 6 weeks, each group of rats was placed on a 1-m tall ladder with uneven intervals. The number of times of rat slips (error steps) was counted and scored with a higher score indicating worse coordination. The total steps of hindlimbs of each rat were recorded as well by two observers. Three independent experiments were performed and the average value was evaluated. The success rate was calculated as follows: success rate $=1-$ slip steps/total steps $\times 100 \%$.

\section{Western blot analysis}

Tissues from the gray matter at the spinal lesion sites were collected and lysed in radio-immunoprecipitation assay lysis buffer (Boster Biological Technology Co., Ltd., Wuhan, Hubei, China), and the protein concentration was determined using a bicinchoninic acid kit (70PQ0012, MultiSciences, Zhejiang, China). Then, the protein was separated on sodium dodecyl sulfatepolyacrylamide gel electrophoresis and transferred to polyvinylidene fluoride membranes. The membranes were blocked with $5 \%$ BSA for $1 \mathrm{~h}$ and then incubated with the primary antibodies to RGMA (1:10000, ab169761, Abcam), NF-200 (1:1000, \#55453, CST), synaptophysin (1:1000, ab14692, Abcam), STAT3 (1: 1000, ab68153, Abcam), p-STAT3 (1:1000, ab32143, Abcam), and glyceraldehyde-3-phosphate dehydrogenase (GAPDH, 1:2000, ab9485, Abcam) at $4{ }^{\circ} \mathrm{C}$ overnight. Next, the membranes were incubated with the horseradish peroxidase (HRP)-labeled goat anti-mouse IgG (1: 2000, ab205719, Abcam) at room temperature for $1 \mathrm{~h}$. The western blots were developed using an enhanced chemiluminescence kit (BB-3501, Amersham Pharmacia Biotech, Piscataway, NJ, USA) exposed by a Gel imager, captured by a Bio-Rad image analysis system (Bio-Rad, Inc., Hercules, CA, USA), and analyzed using the Quantity One v4.6.2 software.

\section{Reverse transcription-quantitative polymerase chain reaction (RT-qPCR)}

Total RNA from tissues was collected using a TRIzol kit (15596026, Invitrogen, Inc., Carlsbad, CA, USA) and reversely transcribed into cDNA using a PrimeScript RT reagent Kit (RR047A, Takara, Holdings Inc., Kyoto, Japan). The cDNA was amplified for qPCR using a Fast SYBR Green PCR Kit (Applied Biosystems, Inc., Carlsbad, CA, USA) on an ABI PRISM 7300 System (Applied biosystems). The primers are shown in Table 1 with U6 as an internal reference for miR-29a and miR-199B while 
Table 1 Primer sequences for RT-qPCR

\begin{tabular}{ll}
\hline Gene & Primer sequence (5'-3') \\
\hline miR-29a & F: GCGCACTGATTTCTTTGGTGTTCAG \\
& R: GCGAGCACAGAATTAATACGAC \\
miR-199B & F: TTATCCTAATTGCTCCTACGGCT \\
& R: ATTCGGCATCGCGCTAAACGTTA \\
RGMA & F: TCACCGACCGCTTCCAGACC \\
& R: CTCCTCACCAGTTACGACACCTC \\
GAPDH & F: GGGAGCCAAAAGGGTCAT \\
& R: GAGTCCTTCCACGATACCAA \\
U6 & F: CGAACGATACAGAGAAGATTAGC \\
& R: CGAACGATACAGAGAAGATTAGC
\end{tabular}

$R T-q P C R$ reverse transcription-quantitative polymerase chain reaction, miR microRNA, RGMA repulsive guidance molecule A, GAPDH glyceraldehyde-3phosphate dehydrogenase, $F$ forward, $R$ reverse

GAPDH for mRNAs. Relative gene expression was determined by the $2^{-\Delta \Delta \mathrm{Ct}}$ method.

\section{Statistical analysis}

SPSS 21.0 (IBM Corp. Armonk, NY, USA) was used for data analysis. Measurement data were expressed as mean \pm standard deviation (SD) from at least three independent experiments. Differences between two groups were analyzed by the $t$ test, while data among multiple groups were compared by one-way or two-way analysis of variance (ANOVA), followed by Tukey's multiple comparison test.

\section{Results}

miR-29a and miR-199B were downregulated in rats with $\mathrm{SCl}$

As mentioned before, miR-29a and miR-199B were reported to be poorly expressed in rat models with SCI and showed protective roles in neuronal recovery. But the detailed functions of these two molecules and the involving mechanisms in SCI remain largely unknown. Here, we established a rat model with SCI. The successful model establishment in rats was confirmed by $<1$ $\mathrm{BBB}$ rating score $1 \mathrm{~h}$ after surgery. According to the RTqPCR results, miR-29a and miR-199B were poorly expressed in the gray matter in rats with SCI compared to the sham-operated ones $(p<0.05)$ (Fig. 1).

Overexpression of miR-29a or miR-199B reduces neuronal injury and promotes the locomotor function of $\mathrm{SCl}$ rats

To explore the functions of miR-29a and miR-199B, miR-29a or miR-199B was overexpressed in model rats through delivering miR-29a/199B mimic. The successful transfection was validated according to the RT-qPCR results (Fig. 2a). Compared to NC mimic, transfection of miR-29a mimic or miR-199B mimic led to a significant increase in miR-29a/199B expression (all $p<0.05$ ).

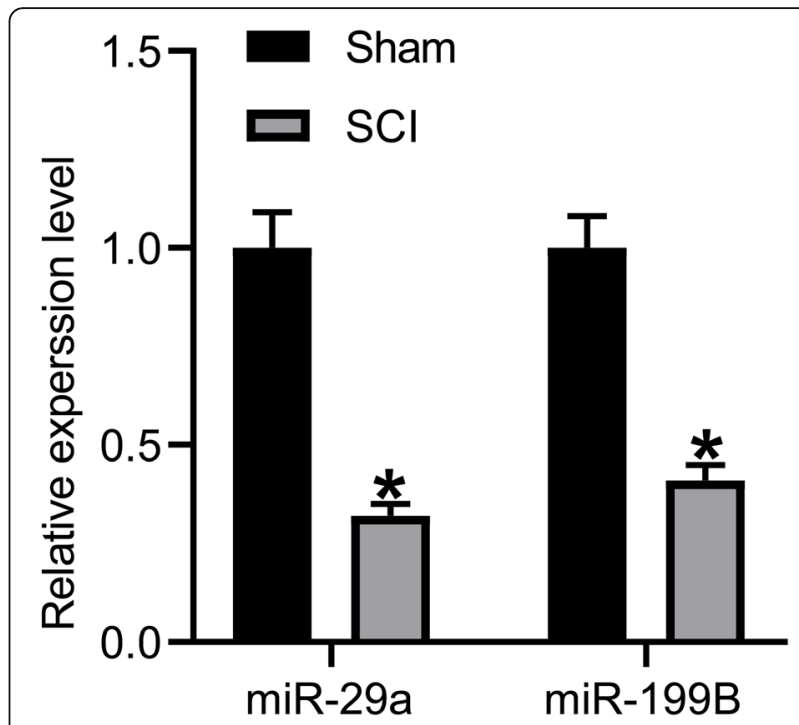

Fig. 1 miR-29a and miR-199B are downregulated in rats with SPI. miR-29a and miR-199B expression in the gray matter in rats determined by RT-qPCR. $N=8$. Data were presented as mean $\pm \mathrm{SD}$ from at least three independent experiments. Data were compared by two-way ANOVA. ${ }^{*} p<0.05$

We then measured the expression of a neurofilament protein NF-200 in the gray matter $2-3 \mathrm{~mm}$ posterior to the injury sites using immunofluorescence staining. It was found that the staining intensity of NF-200 was increased upon miR-29a or miR-199B overexpression (all $p<0.05$ ) (Fig. 2b). In addition, the western blot analysis that the protein levels of NF-200 and synaptophysin were increased after miR-29a and miR-199B upregulation (Fig. 2c).

The neurologic function of rats was monitored weekly using a BBB locomotor rating scale (Fig. 2d). The BBB locomotor scores of rats were significantly increased on the 2nd and 3rd week, respectively, after miR-29a mimic or miR-199B mimic administration (all $p<0.05$ ). A ladder-climbing test was used to evaluate the coordination of rat hindlimbs. It was found the time of slips was notably decreased, and correspondingly, the success step rate was notably increased since the 3rd week after miR29a mimic transfection. Likewise, the error score was reduced while success rate was increased from the 4th week in rats with miR-199B overexpression (all $p<0.05$ ) (Fig. 2e). Collectively, these results suggested that overexpression of miR-29a or miR-199B promotes neurological function recovery in rats with SCI.

\section{miR-29a and miR-199B target RGMA and suppress the} STAT3 signaling pathway

RGMA has been noted to be upregulated in SCI rats and suppressed neurological recovery [10], and STAT3 signaling activation was suggested to inhibit nerve regeneration and repair [21]. Interestingly, either miR-29a or 


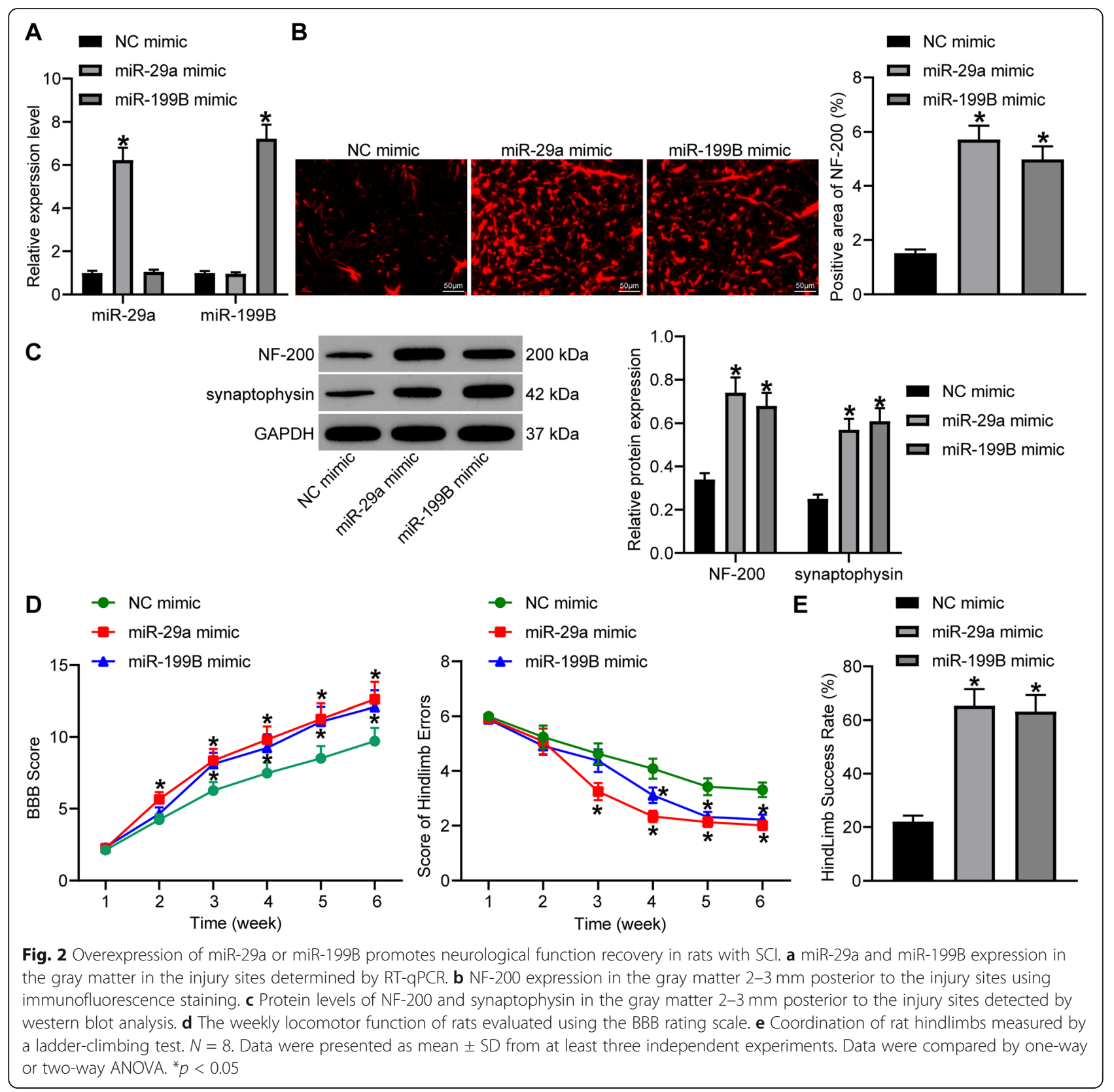

miR-199B was reported to negatively regulate the STAT3 signaling pathway $[22,23]$. Therefore, we determined the protein levels of RGMA and STAT3 as well as the phosphorylation of STAT3 in the tissues in gray matter. The results showed that the protein level of RGMA and the phosphorylation of STAT3 were notably increased in the SCI model rats relative to the shamoperated ones (all $p<0.05$ ), while the total protein level of STAT3 showed little differences between the two groups $(p>0.05)$ (Fig. 3a).

According to the data the bioinformatics system StarBase, we found that either miR-29a or miR-199B had binding sites with RGMA (Fig. 3b, c). Then, the binding relationships between miR-29a/199B and RGMA were further validated through luciferase assays. It was found that co-transfection of miR-29a/199B mimic and pMIRRGMA-WT vector led to a notable decline in luciferase activity in HEK293T cells (all $p<0.05$ ), while the cells transfected with NC mimic or pMIR-RGMA-MUT vector showed little changes in luciferase activity (all $p>$ 0.05 ) (Fig. 3d, e). These results indicated that both miR29a and miR-199B can directly bind to RGMA.

Thereafter, we explored the correlations between the miR-29a/199B expression and the levels of RGMA and STAT3 in the tissues in gray matter. It was found that the protein level of RGMA and phosphorylation of 


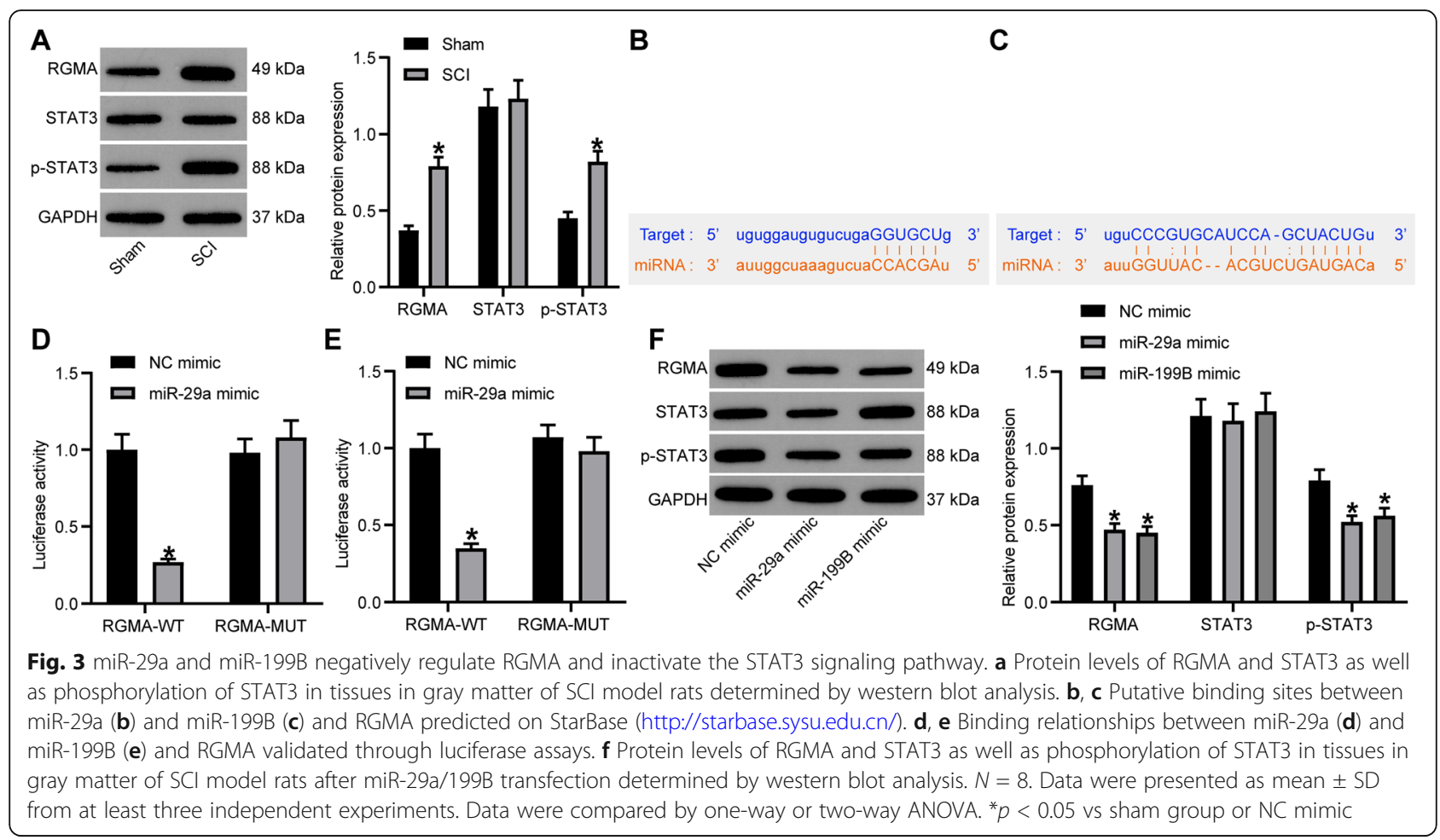

STAT3 were notably decreased after miR-29a mimic and miR-199B mimic transfection in SCI model rats (all $p<0.05)$, but the STAT3 level showed no significant changes $(p>0.05)$ (Fig. 3f).

\section{Silencing of RGMA promotes the neurological recovery in rats following $\mathrm{SCl}$}

To further identify the involvements of RGMA in SCI and its interaction with miR-29a/199B, sh-RGMA and the additional miR-29a/199B inhibitor as well as the corresponding control vectors were administrated into the model rats. According to the RT-qPCR and western blot assays, it was found that the mRNA and protein expression of RGMA was reduced in the tissues in gray matter in SCI rats after sh-RGMA treatment, while the additional downregulation of miR-29a or miR-199B recovered the expression of RGMA in tissues (all $p<0.05$ ) (Fig. 4a, b).

Still, the NF-200 expression in gray matter was determined by immunofluorescence staining. It was found that the staining intensity of NF-200 was notably increased upon RGMA silencing but then reduced by the additional downregulation of miR-29a or miR-199B (all $p<0.05$ ) (Fig. 4c). Similar trends were reproduced in the western blot analysis concerning NF-200 and synaptophysin expression. The protein levels of NF-200 and synaptophysin in tissues were notably enhanced by shRGMA but then reduced by miR-29a or miR-199B inhibitor (all $p<0.05$ ) (Fig. 4d).
Silencing of RGMA suppresses STAT3 signaling pathway to promote the locomotor function recovery in $\mathrm{SCl}$ rats As for the locomotor function in rats, we further found that the $\mathrm{BBC}$ locomotor scores of model rats were notably enhanced after sh-RGMA transfection from the 2nd week following SCI $(p<0.05)$, but the scores were reduced by the additional administration of miR-29a/199B inhibitor since the 3rd week (Fig. 5a). The ladderclimbing test results showed that the number of times of slips (error) was reduced since the 3rd week when RGMA was silenced, companying with an increase in the success step rate. However, the improvements in $\mathrm{BBB}$ locomotor scores and the coordination in rat hindlimbs were blocked by miR-29a/199B inhibitor from the 4th week (all $p<0.05$ ) (Fig. 5b).

We then measured the STAT3 signaling pathway activity in gray matter. It was found that silencing of RGMA led to a significant decline in STAT3 phosphorylation, but this inhibition was then blocked by the further downregulation of miR-29a and miR-199B (all $p<$ $0.05)$. During these processes, the total protein level of STAT3 showed little changes $(p>0.05)$ (Fig. 5c).

\section{Discussion}

SCI has an annual incidence of 10.4-83 million cases globally and bring long-term heavy burdens to patients and the healthy management organizations [24, 25]. miRNAs have emerged as either biomarkers or therapeutic options for SCI management due to their 


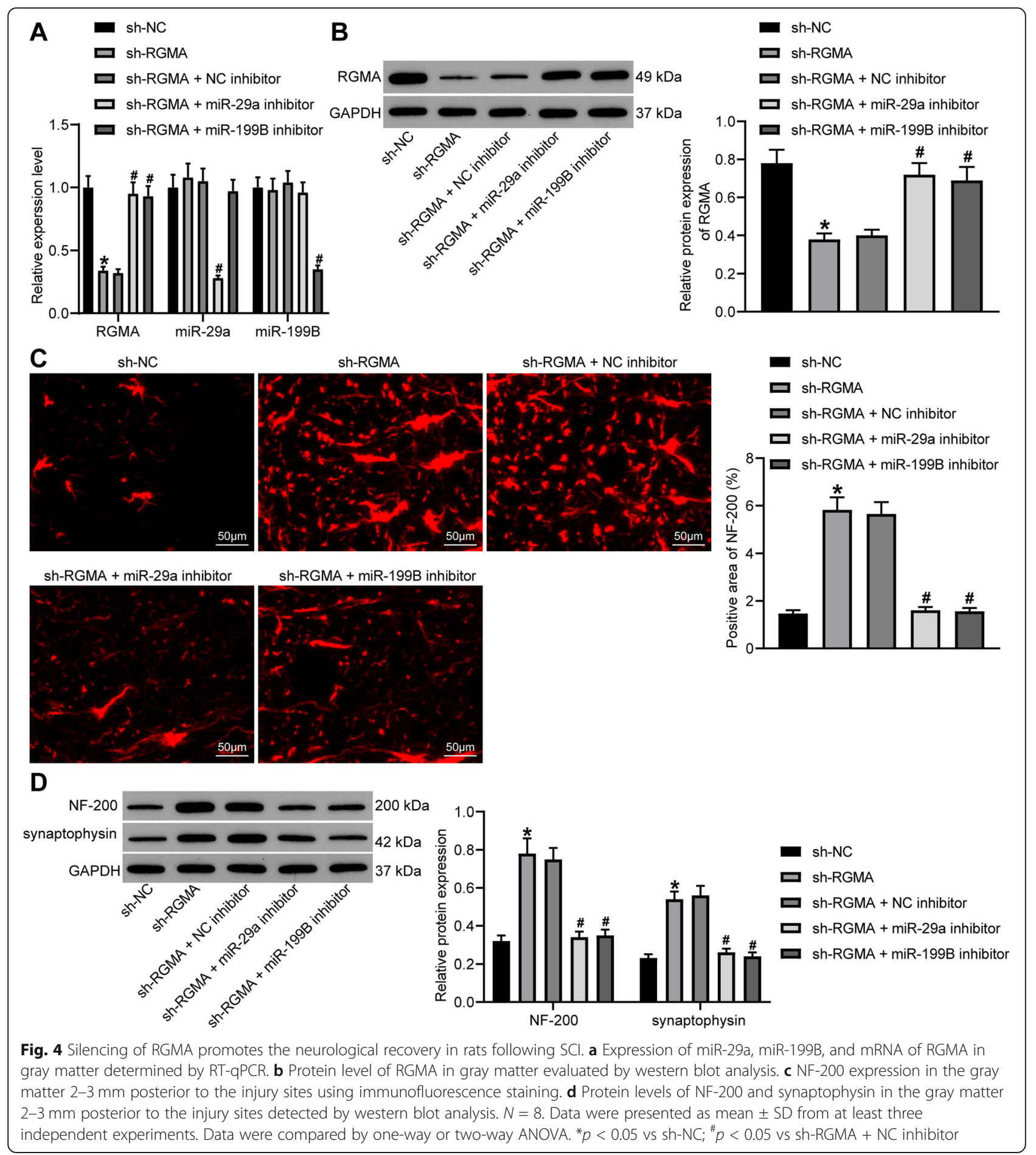

versatile functions in key events such as oligodendrocyte development, axonal regeneration, and remyelination $[7$, 26]. In the present research, we validated the potent functions of miR-29a/199B in neurologic and locomotor functional recovery in a rat model with the involvement of downregulation of RGMA and the suppression of the STAT3 signaling pathway.
After identification of the successful model establishment, the initial finding of the study was that miR-29a and miR-199B were notably downregulated in the SCI model rats, which was in line with the previous studies $[8,9]$. Neural network reconstruction is a key event for functional recovery after SCI. Next, we found that overexpression of miR-29a/199B in the gray matter in lesion 


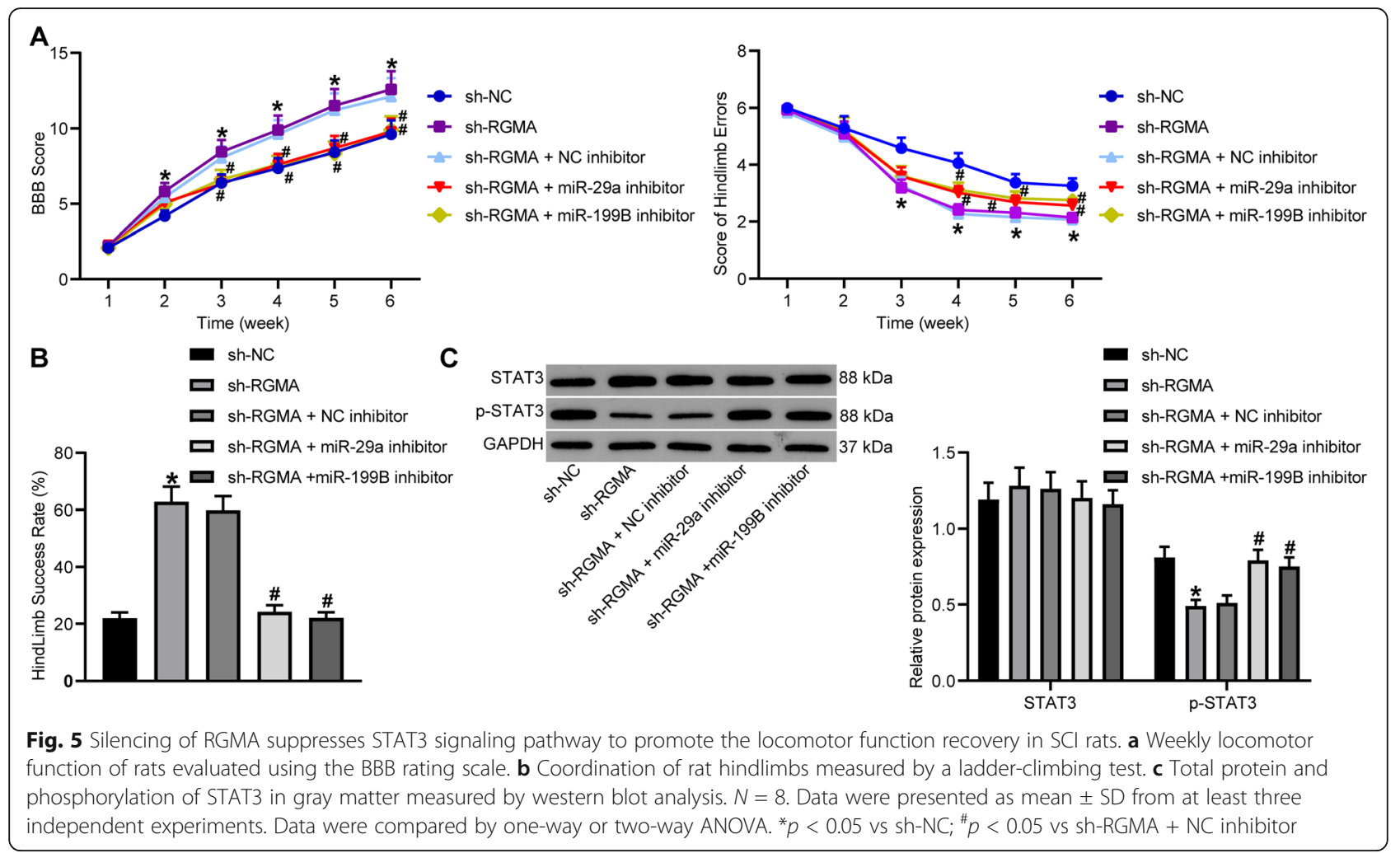

sites led to an increase in the levels of NF-200 and synaptophysin. NF-200 is a neurofilament protein that represents a typical biomarker for mature neuronal cells in the CNS, and thus an effective marker for nerve regeneration [27], while synaptophysin usually serves as an important marker of synaptic density [28]. In addition, the $\mathrm{BBB}$ rating scale suggested that the locomotor score of rats was increased when miR-29a and miR-199B was upregulated. More obviously, the ladder-climbing test suggested that the coordination of rat hindlimb steps was improved upon miR-29a/199B overexpression. The promoting role of miR-29a in nerve regeneration has been well reported. It was reported to regulate axon branching by directly controlling doublecortin in primary neurons [29]. Likewise, miR-29a was found to be upregulated and was possibly responsible for Kuwanon $\mathrm{V}$-induced neurogenesis [30]. miR-29a has been established to have the potency to promote neurite outgrowth in N2a cells [31]. Though miR-199B is relatively less concerned, it has been noted to be poorly expressed and suppress hypoxia-inducible factor-1 alpha to counter the progression of human intractable epilepsy [32]. Here, we validated the neurological recovery promotion of these two miRNAs.

miRNAs exert their functions primarily by binding to the diverse downstream mRNAs. Here, the integrated mRNA prediction on StarBase system and the dualluciferase reporter gene assays validated that miR-29a and miR-199B shared a binding relationship with RGMA. Then, we found RGMA was upregulated in the gray matter in lesion sites in model rats. RGMA is a potent suppressor of axon regeneration and neurite outgrowth [33]. It was reported to express in the glial scar and myelin and is accumulated in lesioned sites after a traumatic injury of the spinal cord and brains in rodents [10]. In addition to rodents, administration of neutralizing antibody against RGMA was found to lead to recovery from impaired manual dexterity in a monkey model of SCI [12]. On this basis, we further modified RGMA expression in the SCI rat models. Consequently, artificial downregulation of RGMA led to an increase in the levels of NF-200 and synaptophysin, and the locomotor function and coordination of rats were improved. However, the promoting roles of sh-RGMA were blocked by the further downregulation of miR-29a and miR-199B, indicating that silencing of RGMA is accountable for miR29a/199B-mediated neurologic function recovery.

Either miR-29a or miR-199B has been reported to regulate the STAT3 signaling pathway $[22,23]$, which is a well-known transcription factor especially participating in cell proliferation and inflammation in the lesion site after SCI, including inducing astrocyte proliferation, astrogliosis, and scar formation [15, 34]. Similarly, activation of STAT3 has been demonstrated to suppress nerve regeneration and repair and induce neuropathic pain and inflammation [21, 35, 36]. Here, our study found that 
phosphorylation of STAT3 was increased in the SCI model rats, and miR-29a or miR-199B upregulation was found to suppress this trend. Interestingly, silencing of RGMA was also observed to suppress the STAT3 phosphorylation, indicating that STAT3 activation is possibly implicated in RGMA-mediated nerve degeneration inhibition.

\section{Conclusion}

To conclude, our study evidenced that miR-29a/199B can promote neurologic recovery and improve the locomotor function of rats with SCI through suppressing RGMA expression, which leads to further downregulation of STAT3. However, there remains a major limitation in this research that although we evidenced RGMA silencing suppresses STAT3 phosphorylation, the detailed regulatory network and the exact involvement of STAT3 in RGMA mediation need further evidence. We would like to perform rescue experiments to validate the interactions between RGMA and STAT3 phosphorylation and their joint effects on neuronal growth in the near future. We also hope more studies will be conducted in this field to provide more understandings in the molecular mechanism in SCI and offer more ideas for SCI control.

\begin{abstract}
Abbreviations
ANOVA: Analysis of variance; BBB: Basso, Beattie, and Bresnahan; CNS: Central nervous system; GAPDH: Glyceraldehyde-3-phosphate dehydrogenase; HRP: Horseradish peroxidase; IgG: Immunoglobulin G; LV: Lentiviral vector; mean \pm SD: Mean \pm standard deviation; miRNAs: MicroRNAs; MUT: Mutant type; NC: Negative control; RGMA: Repulsive guidance molecule A; RTqPCR: Reverse transcription-quantitative polymerase chain reaction; SCl: Spinal cord injury; shRNA: Short hairpin RNA; SPF: Specific-pathogen-free; STAT3: Signal transducer and activator of transcription 3; WT: Wild-type
\end{abstract}

\section{Acknowledgements}

Not applicable.

\section{Authors' contributions}

All authors made substantial contributions to conception and design, acquisition of data, or analysis and interpretation of data; took part in drafting the article or revising it critically for important intellectual content; gave final approval of the version to be published; and agree to be accountable for all aspects of the work

\section{Funding}

Not applicable.

\section{Availability of data and materials}

All the data generated or analyzed during this study are included in this published article.

\section{Ethics approval and consent to participate}

The study was performed with the approval of the Ethical Committee of Shanghai Eighth People's Hospital (Approval No. 2019011). All experimental procedures were conducted in line with the ethical guidelines for the study of experimental pain in conscious animals. Great efforts were made to minimize the usage and suffering of animals.

\section{Consent for publication}

Not applicable.

\section{Competing interests}

The authors declare that they have no competing interests.

Received: 23 July 2020 Accepted: 10 September 2020

Published online: 18 September 2020

\section{References}

1. Freund P, Seif M, Weiskopf N, Friston K, Fehlings MG, Thompson AJ, Curt A. MRI in traumatic spinal cord injury: from clinical assessment to neuroimaging biomarkers. Lancet Neurol. 2019;18:1123-35. https://doi.org/ 10.1016/S1474-4422(19)30138-3.

2. Eckert MJ, Martin MJ. Trauma: spinal cord injury. Surg Clin North Am. 2017; 97:1031-45. https://doi.org/10.1016/j.suc.2017.06.008.

3. Li HL, Xu H, Li YL, Sun SW, Song WY, Wu Q, Ai J, Sun JC, Ning GZ, Feng SQ. Epidemiology of traumatic spinal cord injury in Tianjin, China: An 18-year retrospective study of 735 cases. J Spinal Cord Med. 2019;42:778-85. https:// doi.org/10.1080/10790268.2017.1415418.

4. Snyder R, Verla T, Ropper AE. Practical application of recent advances in diagnostic, prognostic, and therapeutic modalities for spinal cord injury. World Neurosurg. 2020;136:330-6. https://doi.org/10.1016/j.wneu.2020.01. 011 .

5. Tigchelaar S, Streijger F, Sinha S, Flibotte S, Manouchehri N, So K, Shortt K, Okon E, Rizzuto MA, Malenica I, Courtright-Lim A, Eisen A, Keuren-Jensen KV, Nislow C, Kwon BK. Serum microRNAs reflect injury severity in a large animal model of thoracic spinal cord injury. Sci Rep. 2017;7:1376. https://doi. org/10.1038/s41598-017-01299-x.

6. Ning B, Gao L, Liu RH, Liu Y, Zhang NS, Chen ZY. microRNAs in spinal cord injury: potential roles and therapeutic implications. Int J Biol Sci. 2014;10: 997-1006. https://doi.org/10.7150/ijbs.9058.

7. Shi Z, Zhou H, Lu L, Li X, Fu Z, Liu J, Kang Y, Wei Z, Pan B, Liu L, Kong X, Feng S. The roles of microRNAs in spinal cord injury. Int J Neurosci. 2017; 127:1104-15. https://doi.org/10.1080/00207454.2017.1323208.

8. Yin H, Shen L, Xu C, Liu J. Lentivirus-Mediated Overexpression of miR-29a promotes axonal regeneration and functional recovery in experimental spinal cord injury via PI3K/Akt/mTOR pathway. Neurochem Res. 2018;43: 2038-46. https://doi.org/10.1007/s1 1064-018-2625-5.

9. Zhou HJ, Wang LQ, Xu QS, Fan ZX, Zhu Y, Jiang H, Zheng XJ, Ma YH, Zhan RY. Downregulation of miR-199b promotes the acute spinal cord injury through IKKbeta-NF-kappaB signaling pathway activating microglial cells. Exp Cell Res. 2016;349:60-7. https://doi.org/10.1016/j.yexcr.2016.09.020

10. Mothe AJ, Tassew NG, Shabanzadeh AP, Penheiro R, Vigouroux RJ, Huang L, Grinnell C, Cui YF, Fung E, Monnier PP, Mueller BK, Tator CH. RGMa inhibition with human monoclonal antibodies promotes regeneration, plasticity and repair, and attenuates neuropathic pain after spinal cord injury. Sci Rep. 2017;7:10529. https://doi.org/10.1038/s41598-017-10987-7.

11. Chen J, Shifman MI. Inhibition of neogenin promotes neuronal survival and improved behavior recovery after spinal cord injury. Neuroscience. 2019;408: 430-47. https://doi.org/10.1016/j.neuroscience.2019.03.055.

12. Nakagawa H, Ninomiya T, Yamashita T, Takada M. Treatment with the neutralizing antibody against repulsive guidance molecule-a promotes recovery from impaired manual dexterity in a primate model of spinal cord injury. Cereb Cortex. 2019;29:561-72. https://doi.org/10.1093/cercor/bhx338.

13. Cui M, Ma X, Sun J, He J, Shen L, Li F. Effects of STAT3 inhibitors on neural functional recovery after spinal cord injury in rats. Biosci Trends. 2017;10: 460-6. https://doi.org/10.5582/bst.2016.01160.

14. Zong S, Zeng G, Fang Y, Peng J, Tao Y, Li K, Zhao J. The role of IL-17 promotes spinal cord neuroinflammation via activation of the transcription factor STAT3 after spinal cord injury in the rat. Mediat Inflamm. 2014;2014: 786947. https://doi.org/10.1155/2014/786947.

15. Herrmann JE, Imura T, Song B, Qi J, Ao Y, Nguyen TK, Korsak RA, Takeda K, Akira S, Sofroniew MV. STAT3 is a critical regulator of astrogliosis and scar formation after spinal cord injury. J Neurosci. 2008;28:7231-43. https://doi. org/10.1523/JNEUROSCl.1709-08.2008.

16. Kjell J, Olson L. Rat models of spinal cord injury: from pathology to potential therapies. Dis Model Mech. 2016;9:1125-37. https://doi.org/10.1242/dmm. 025833.

17. Mattucci S, Speidel J, Liu J, Kwon BK, Tetzlaff W, Oxland TR. Basic biomechanics of spinal cord injury - how injuries happen in people and how animal models have informed our understanding. Clin Biomech (Bristol, Avon). 2019;64:58-68. https://doi.org/10.1016/..clinbiomech.2018.03. 020. 
18. Hodgetts SI, Yoon JH, Fogliani A, Akinpelu EA, Baron-Heeris D, Houwers IGJ, Wheeler LPG, Majda BT, Santhakumar S, Lovett SJ, Duce E, Pollett MA, Wiseman TM, Fehily B, Harvey AR. Cortical AAV-CNTF gene therapy combined with intraspinal mesenchymal precursor cell transplantation promotes functional and morphological outcomes after spinal cord injury in adult rats. Neural Plast. 2018;2018:9828725. https://doi.org/10.1155/2018/ 9828725.

19. Zhou H, Yin C, Zhang Z, Tang H, Shen W, Zha X, Gao M, Sun J, Xu X, Chen Q. Proanthocyanidin promotes functional recovery of spinal cord injury via inhibiting ferroptosis. J Chem Neuroanat. 2020;107:101807. https://doi.org/ 10.1016/j.jchemneu.2020.101807.

20. All AH, Al Nashash H, Mir H, Luo S, Liu X. Characterization of transection spinal cord injuries by monitoring somatosensory evoked potentials and motor behavior. Brain Res Bull. 2020;156:150-63. https://doi.org/10.1016/j. brainresbull.2019.12.012.

21. Fu XM, Wang Y, Fu WL, Liu DH, Zhang CY, Wang QL, Tong XJ. The combination of adipose-derived Schwann-like cells and acellular nerve allografts promotes sciatic nerve regeneration and repair through the JAK2/ STAT3 signaling pathway in rats. Neuroscience. 2019:422:134-45. https://doi. org/10.1016/j.neuroscience.2019.10.018.

22. Gao J, Shao Z, Yan M, Fu T, Zhang L, Yan Y. Targeted regulationof STAT3 by miR-29a in mediating Taxol resistance of nasopharyngeal carcinoma cell line CNE-1. Cancer Biomark. 2018;22:641-8. https://doi.org/10.3233/CBM170964.

23. Peng W, He D, Shan B, Wang J, Shi W, Zhao W, Peng Z, Luo Q, Duan M, Li B, Cheng Y, Dong Y, Tang F, Zhang C, Duan C. LINC81507 act as a competing endogenous RNA of miR-199b-5p to facilitate NSCLC proliferation and metastasis via regulating the CAV1/STAT3 pathway. Cell Death Dis. 2019;10:533. https://doi.org/10.1038/s41419-019-1740-9.

24. Karsy M, Hawryluk G. Modern medical management of spinal cord injury. Curr Neurol Neurosci Rep. 2019;19:65. https://doi.org/10.1007/s11910-0190984-1.

25. Ong B, Wilson JR, Henzel MK. Management of the patient with chronic spinal cord injury. Med Clin North Am. 2020;104:263-78. https://doi.org/10. 1016/j.mena.2019.10.006.

26. Li $\mathrm{P}$, Teng $\mathrm{ZQ}$, Liu CM. Extrinsic and intrinsic regulation of axon regeneration by microRNAs after spinal cord injury. Neural Plast. 2016;2016:1279051. https://doi.org/10.1155/2016/1279051.

27. He B, Nan G. Neuronal regeneration after acute spinal cord injury in adult rats. Spine J. 2016;16:1459-67. https://doi.org/10.1016/j.spinee.2016.06.020.

28. Orczykowski ME, Arndt KR, Palitz LE, Kramer BC, Pessina MA, Oblak AL, Finklestein SP, Mortazavi F, Rosene DL, Moore TL. Cell based therapy enhances activation of ventral premotor cortex to improve recovery following primary motor cortex injury. Exp Neurol. 2018;305:13-25. https:// doi.org/10.1016/j.expneurol.2018.03.010.

29. Li H, Mao S, Wang H, Zen K, Zhang C, Li L. MicroRNA-29a modulates axon branching by targeting doublecortin in primary neurons. Protein Cell. 2014; 5:160-9. https://doi.org/10.1007/s13238-014-0022-7.

30. Kong SY, Park MH, Lee M, Kim JO, Lee HR, Han BW, Svendsen CN, Sung SH, Kim HJ. Kuwanon V inhibits proliferation, promotes cell survival and increases neurogenesis of neural stem cells. PLoS One. 2015;10:e0118188. https://doi.org/10.1371/journal.pone.0118188.

31. Tan C, Yu C, Song Z, Zou H, Xu X, Liu J. Expression of microRNA-29a regulated by yes-associated protein modulates the neurite outgrowth in N2a cells. Biomed Res Int. 2017;2017:5251236. https://doi.org/10.1155/2017/ 5251236.

32. Jiang G, Zhou R, He X, Shi Z, Huang M, Yu J, Wang X. Expression levels of microRNA-199 and hypoxia-inducible factor-1 alpha in brain tissue of patients with intractable epilepsy. Int J Neurosci. 2016;126:326-34. https:// doi.org/10.3109/00207454.2014.994209.

33. Hata K, Fujitani M, Yasuda Y, Doya H, Saito T, Yamagishi S, Mueller BK, Yamashita T. RGMa inhibition promotes axonal growth and recovery after spinal cord injury. J Cell Biol. 2006;173:47-58. https://doi.org/10.1083/jcb. 200508143.

34. Chen X, Chen C, Hao J, Zhang J, Zhang F. Effect of CLIP3 upregulation on astrocyte proliferation and subsequent glial scar formation in the rat spinal cord via STAT3 pathway after injury. J Mol Neurosci. 2018;64:117-28. https:// doi.org/10.1007/s12031-017-0998-6.

35. Dai J, Yu GY, Sun HL, Zhu GT, Han GD, Jiang HT, Tang XM. MicroRNA-210 promotes spinal cord injury recovery by inhibiting inflammation via the
JAK-STAT pathway. Eur Rev Med Pharmacol Sci. 2018;22:6609-15. https://doi org/10.26355/eurrev_201810_16135.

36. Liu Y, Feng L, Ren S, Zhang Y, Xue J. Inhibition of IncRNA DILC attenuates neuropathic pain via the SOCS3/JAK2/STAT3 pathway. Biosci Rep. 2020;40. https://doi.org/10.1042/BSR20194486.

\section{Publisher's Note}

Springer Nature remains neutral with regard to jurisdictional claims in published maps and institutional affiliations.

\section{Ready to submit your research? Choose BMC and benefit from:}

- fast, convenient online submission

- thorough peer review by experienced researchers in your field

- rapid publication on acceptance

- support for research data, including large and complex data types

- gold Open Access which fosters wider collaboration and increased citations

- maximum visibility for your research: over $100 \mathrm{M}$ website views per year

At BMC, research is always in progress.

Learn more biomedcentral.com/submissions 